\title{
Electronic states in an atomistic carbon quantum dot patterned in graphene
}

\author{
L. Craco, ${ }^{1}$ S. S. Carara, ${ }^{1}$ T. A. da Silva Pereira, ${ }^{1}$ and M. V. Milošević ${ }^{2}$ \\ ${ }^{1}$ Instituto de Física, Universidade Federal de Mato Grosso, 78060-900, Cuiabá, MT, Brazil \\ ${ }^{2}$ Departement Fysica, Universiteit Antwerpen, Groenenborgerlaan 171, B-2020 Antwerpen, Belgium \\ (Received 10 September 2015; revised manuscript received 29 February 2016; published 12 April 2016)
}

\begin{abstract}
We reveal the emergence of metallic Kondo clouds in an atomistic carbon quantum dot, realized as a single-atom junction in a suitably patterned graphene nanoflake. Using density functional dynamical mean-field theory (DFDMFT) we show how correlation effects lead to striking features in the electronic structure of our device, and how those are enhanced by the electron-electron interactions when graphene is patterned at the atomistic scale. Our setup provides a well-controlled environment to understand the principles behind the orbital-selective Kondo physics and the interplay between orbital and spin degrees of freedom in carbon-based nanomaterials, which indicate new pathways for spintronics in atomically patterned graphene.
\end{abstract}

DOI: 10.1103/PhysRevB.93.155417

\section{INTRODUCTION}

Graphene, a two-dimensional network of carbon atoms arranged in a hexagonal lattice [1], is attracting the attention of the wider scientific community due to its potential application in fields as diverse as photonics, sensor technology, and spintronics [2]. In fact, graphene and its derivatives [3] are expected to form the next generation of (radio frequency) transistors [4], spin-based [5] and nanoelectronic [6] devices, as well as electrochemical and biosensors [2]. However, the semimetallic nature of graphene with a Dirac-like spectrum near the Fermi energy $\left(E_{F}\right)$ and finite conductivity values [7] prevent its application as the host material for future spintronic applications [8]. Essentially, what is needed for having useful spintronic devices made of graphene or functionalized graphene is to have the electronic spectra similar to those of classical diluted magnetic semiconductors [9].

After several years of study it is now understood that the physical properties of graphene at low energies are mostly governed by elementary excitations created around the Fermi surface [10]. These elementary excitations are known to be massless Dirac fermions with a linear spectrum. On the other hand, at graphene edges the density of states (DOS) may be peaked due to the presence of edge-localized states [11]. At extended zigzag edges this leads to unconventional magnetism, which is caused by ferromagnetic intraedge and antiferromagnetic interedge correlations [12]. Interestingly, to understand the origin of edge magnetism, the importance of electronelectron interactions at edges of chiral graphene nanoribbons was addressed experimentally [13]. However, much of the existing literature on graphene-based systems has nevertheless focused on noninteracting electrons moving on a honeycomb lattice. The main reason for this is that electron-electron interactions are suppressed close to the charge neutrality point because of the vanishing DOS near $E_{F}$ [14]. Hence, electronic correlation effects were often considered to be of minor importance in graphene.

The possibility of correlated electron physics in carbonbased materials $[15,16]$ or in purely $p$ - [17] and $s$ - [18] band systems remains intriguing, since the naive expectation dictates that the itinerance (kinetic energy of $p, s$ carriers) is appreciable compared to the electron-electron interactions, as distinct from $d$-band systems, where the $d$ electrons reside in much narrower bands. Hence, the effective ratio between the on-site Coulomb repulsion and the one-particle bandwidth $(U / W)$ is sizable [19]. This controversy makes the possible relevance of intrinsic electron-electron interactions in graphene [20] or in carbon-based materials with active $p$ bands an issue of contemporary interest. With this in mind, in this work we show how the interplay between finite size localization and sizable on-site Coulomb repulsion [21] can induce orbital-selective Kondo-like phenomenon [14] in a carbon quantum dot made of atomistically engineered graphene.

The Kondo effect is usually induced by the presence of a dilute concentration of localized spins coupled to a Fermi sea of electrons in conventional Fermi liquid metals [22]. There, dynamical many-particle interactions between the spin of the impurity and the electron in the reservoir result in partial screening of the localized spin, leading to a sharp Kondo quasiparticle resonance in the one-particle spectral function [23]. Recent advances in quantum dots [23] and atomic physics [24] have provided new ways to probe and understand Kondo-like phenomena in real mesoscopic quantum systems $[25,26]$. Most of the early theoretical studies on Kondo effect were carried out for conventional metals with idealized DOS near $E_{F}$ [23]. However, in spite of many studies, there has been no discussion to date on the possibility of a multiorbital Kondo cloud in an atomistic carbon quantum dots patterned out of graphene. Here, we shed light onto origins and consequences of this problem of fundamental importance by revealing channel-selective Kondo physics and incipient local moment formation in atomistic carbon quantum dot as a junction between two charge neutral trigonal zigzag graphene flakes [27,28]. More precisely, the initial schematic structure we use to investigate electronic and magnetic responses of a single carbon quantum dot is shown in the top panel of Fig. 1 and represents a pattern $[29,30]$ obtained by suitably cutting a hydrogenated graphene zigzag nanoribbon.

\section{RESULTS AND DISCUSSION}

It is noteworthy that in engineered nanostructures the hopping elements are renormalized in rather nontrivial ways [31]. On the other hand, due to its atomistic nature the on-site 

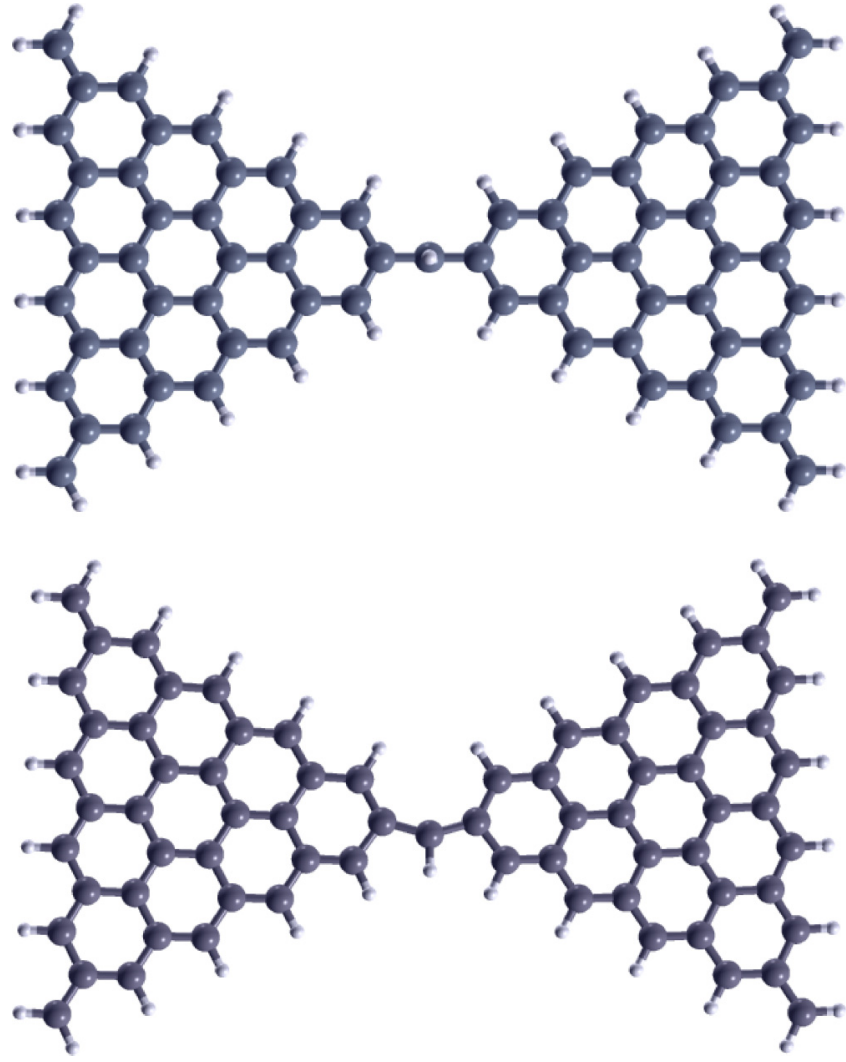

FIG. 1. Top views of an atomistic carbon quantum dot at a junction patterned between two graphene nanoflakes. The initial schematic (upper panel) and the optimized geometrical structure (lower panel) are shown in the same figure for comparison. Carbon and hydrogen atoms are shown in dark and light colors, respectively. Notice that in the optimized geometry a small in-plane deformation is found, which is manifested in the bending of the central atom to its nearest carbon atoms.

Coulomb $U$ interaction is expected to be less affected by edge shapes and nanoscale. With this in mind, we examine the effect of electron-electron interactions on the electronic structure of our atomistic carbon quantum dot, showing how it can be reshaped by realistic multiorbital interaction effects. Here, optimum geometry, stability and one-particle electronic properties of the system were investigated within density functional theory (DFT) as implemented in the SIESTA simulation package [32]. To ensure that there is no interaction between successive periodic images, we use a cell with vacuum space in all directions. The respective Brillouin zone is sampled by a $10 \times 10 \times 2$ Monkhorst-Pack grid [33]. The geometry was fully optimized until all the force components became smaller than $0.05 \mathrm{eV} / \AA$. Norm-conserving pseudopotentials of Troullier-Martins [34] in Kleinman-Bylander nonlocal form were used to represent the ionic core potential. We use the generalized gradient approximation (GGA) scheme, as parameterized by Perdew et al. [35], for the exchange-correlation functional. The Kohn-Sham orbitals [36] are expanded in a linear combination of atomic orbitals of finite range, which is determined by a common confinement energy shift of 0.01 Ry [37]. The precision of the real-space grid integration is determined by a minimal energy cutoff of $200 \mathrm{Ry}$ [38].

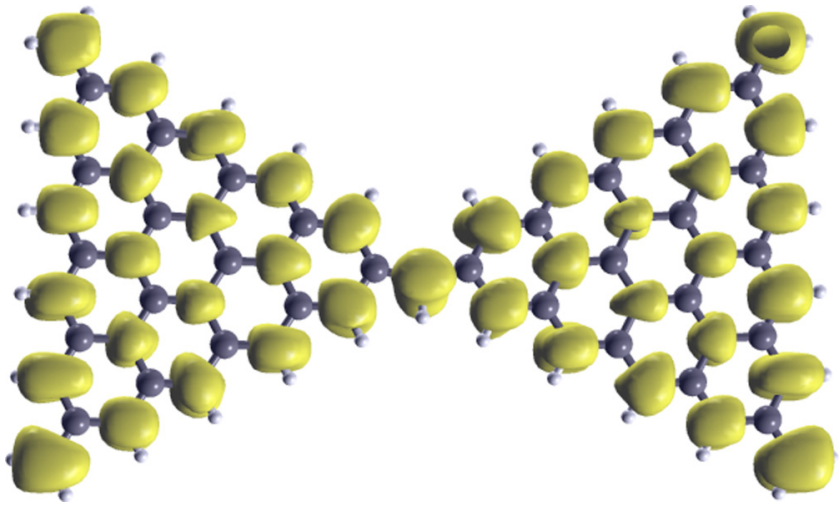

FIG. 2. The spin density isosurfaces $\left(3 \times 10^{-3} e / \AA^{3}\right)$ in the most stable structural and spin configuration of an atomistic carbon quantum dot at a junction patterned out between two graphene nanoflakes. The spin density isosurfaces show that the net spin density is mostly located at a particular site of the optimized geometrical structure.

\section{A. GGA results}

We now present our GGA results. To begin with, we show in Fig. 1 (lower panel) the optimized geometrical structure of our carbon nanosystem. Consistent with previous studies [29,39] on triangular carbon nanoflakes, our optimized geometry shows a small in-plane deformation configuration [39] which is manisfested in the bending angle of the central atom and its nearest $\mathrm{C}$ atoms. Indeed, our optimized geometrical structure reveals that the calculated angle between our linking carbon quantum dot and its nearest two $\mathrm{C}$ atoms changes from $180^{\circ}$ to $163.73^{\circ}$ (see Fig. 1). This result is consistent with geometric optimizations carried out by $\mathrm{Li}$ et al. [29] for a low-dimensional structure with similar carbon building blocks. Also, in good agreement with previous studies [29,30] are the spin density isosurfaces (see Fig. 2), showing that the net spin density is mostly located at $A$ sites [29] of the hexagonal ( $A-B)$ structure, where the spin densities on $B$ sites are rather small.

To highlight the spin-polarized electronic nature [29,39] of our optimized geometrical structure, in Fig. 3 we show the spin- and orbital-resolved GGA spectral functions of the central carbon quantum dot. It is known that in graphene, the $s p^{2}$ hybridization of atomic $s-p_{x, y}$ orbitals of carbon atoms creates lateral $\sigma$ bonds, and the remaining $2 p_{z}$ orbital perpendicular to the plain form the nonhybridized $\pi$ bands. This well-established scenario for graphene and graphite [40] dramatically changes in our mesoscopic quantum system. As seen in Fig. 3, while the planar $p_{x}$ DOS shows large bonding-antibonding splitting, the semiconductinglike band gap within the $p_{y}$ orbitals is considerably reduced and localized electronic states are formed near $E_{F}$ due to finite overlap with the $p_{z}$ channel. Moreover, the atomically resolved DOS of the $p_{z}$ orbital does not vanish linearly near the Fermi energy $\left(\omega=E_{F}=0\right)$, exhibiting instead a small gap at low energies. Also interesting are the localized states (almost flat bands) in the spin-resolved $p_{y, z}$ spectral functions near $E_{F}$, which as shown below are the one-particle seeds towards the formation of an orbital-selective Kondo state at finite values of the on-site Coulomb repulsion $U$. Noteworthy, our spin-resolved electronic structure in Fig. 3 is consistent 


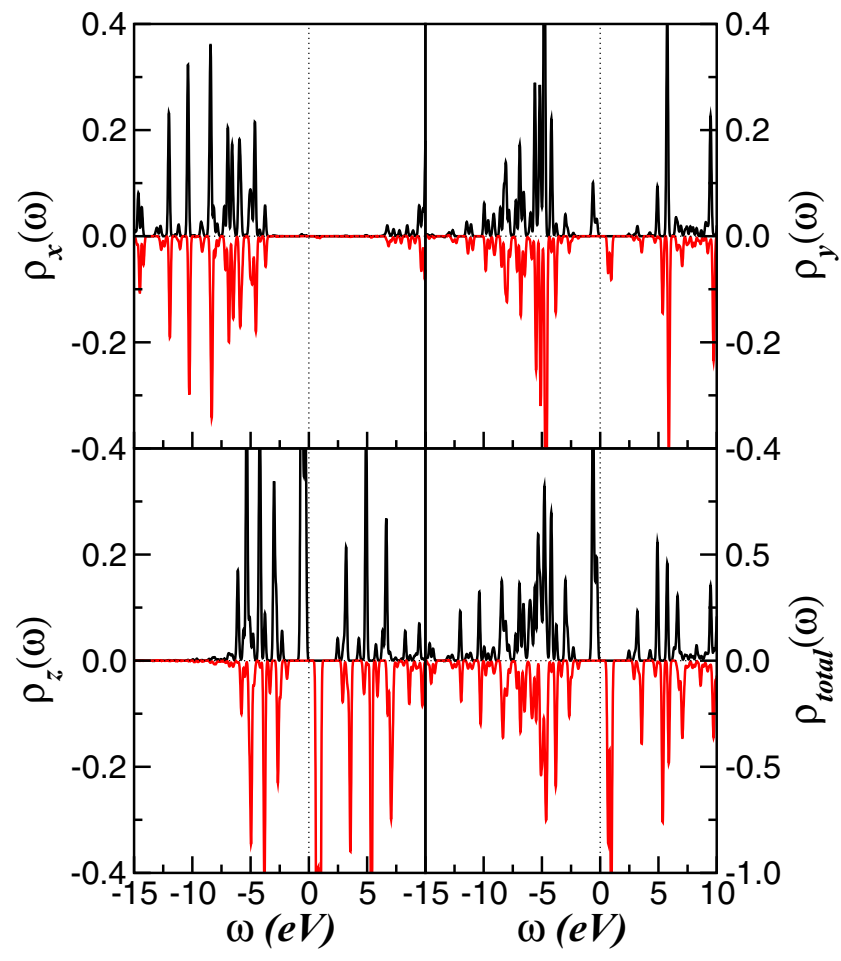

FIG. 3. GGA orbital- and spin-resolved density of states (DOS) of our carbon quantum dot (i.e., the central atom in Fig. 1). Notice the spin polarization caused by zero-energy edge states and the large bonding-antibonding band gap within the $p_{x}$ orbital. A particular feature to note is the small band gap in the $p_{y, z}$ channels and the narrow bands near the Fermi level, which are the one-particle seeds towards the formation of channel-selective Kondo clouds.

with previous calculations on graphene nanoflakes [41], supporting the existence of spontaneous magnetic ordering in triangular graphene nanoflakes linked by different atoms [29] or by carbon chains containing an odd (even) number of $\mathrm{C}$ atoms [30]. In fact, after several years of study it is now recognized that magnetism in pure carbon materials is closely related to localized edge states which creates almost flat bands near $E_{F}$ [42], favoring spin polarization. Of particular interest here is the case where two graphene nanoflakes are linked by a single carbon atom $[29,30]$, showing large total magnetic moment and spontaneous ferromagnetic coupling between the nanoflakes. In good agreement with previous studies $[29,30]$, our GGA results confirm that when two hydrogenated triangular nanoflakes [39] are linked by a single carbon atom, the ferromagnetic ground state (with large total magnetic moment) [29] is favored compared to the antiferromagnetically ordered state, in accordance with Lieb's theorem [43]. Taken together, this gives us confidence to use the GGA DOS of Fig. 3 as an input to multiorbital GGA+DMFT calculations for a carbon quantum dot patterned in graphene.

\section{B. GGA+DMFT: Theory and results}

Electron-electron interactions and multiorbital correlations are intricately tied to lattice, charge, and spin degrees of freedom in correlated electron systems [19]. Theoretically, we are still away from being able to treat these coupled correlations in a fully realistic manner, particularly for multiorbital quantum dots where the hidden many-body problem remains an interesting and open issue, both theoretically and experimentally [25]. Precisely how this might come about is an open and challenging problem also for wide band systems. Here, we study correlation-induced electronic reconstruction using combined DFT and DMFT methods applied to a real carbon quantum dots. A similar scheme for lattice systems was used to revisit the longstanding issues of transport anisotropy and incoherence-coherence crossovers in graphite [40], as well as in the context of Kondo physics in strained graphene [14]. This justifies the use of density functional dynamical meanfield theory (DFDMFT) to study the electronic properties of a real carbon quantum dot coupled to electronic reservoirs.

The bare and partially screened Coulomb interaction parameters for pure graphene are, respectively, $U=17.0 \mathrm{eV}$ and $U=9.3 \mathrm{eV}$ [21]. Owing to the insulating nature of our system at the one-particle level (see Fig. 3), one can naively expect the Hubbard $U$ (or the on-site Coulomb interaction) of the carbon quantum dot to be rather poorly screened as compared to good metals. In our study we choose renormalized $U$ values to reveal an interaction-induced Kondo phenomena, which are characterized by the appearance of orbital-selective Kondo resonances near $E_{F}$ at finite $U$ values. The many-body Hamiltonian considered here is $H=H_{0}+H_{\text {int }}$, with

$$
H_{0}=\sum_{\mathbf{k} a \sigma} \varepsilon_{a}(\mathbf{k}) c_{\mathbf{k} a \sigma}^{\dagger} c_{\mathbf{k} a \sigma}
$$

and

$$
H_{\text {int }}=U \sum_{a} n_{a \uparrow} n_{a \downarrow}+\sum_{a \neq b} U^{\prime} n_{a} n_{b}-J_{H} \sum_{a \neq b} \mathbf{S}_{a} \cdot \mathbf{S}_{b},
$$

where $a=x, y, z$ label the diagonalized $p$ bands. $\epsilon_{a}(\mathbf{k})$ is the one-electron band dispersion projected on the central atom, which encodes details of its actual one-electron (GGA) band structure as well as its hybridization with the two neighboring trigonal graphene nanoflakes. $U^{\prime} \equiv U-2 J_{H}$, with $U, U^{\prime}$ being the atomic [44] intra- and interorbital Coulomb repulsion and $J_{H}$ is the Hund's rule coupling [14,40]. Here, the peculiar one-particle nature of the multichannel electronic states in our carbon quantum dot are read off from the projected DOS shown in Fig. 3, which are DFT inputs for the multiorbital DMFT approach that reveals a selective Kondo state at low energies.

To reveal correlation-induced electronic reconstruction in our nanoembedded carbon atom, we extend ideas based on real-space DMFT [45]. In real-space DMFT each lattice site of a nanosystem is mapped onto its own impurity model. However, as in the usual DMFT treatment [46] this mapping is done by computing the local Green's function at each site via

$$
G_{\sigma}^{\mathrm{loc}}(\omega)=\frac{1}{N} \sum_{\mathbf{k}} \frac{1}{\omega-\Sigma_{\sigma}(\omega)-\varepsilon_{\mathbf{k}}},
$$

where $\varepsilon_{\mathbf{k}}$ is the one-electron band dispersion of a chosen lattice and $\Sigma_{\sigma}(\omega)$ is the corresponding momentum-independent selfenergy, which encodes all many-particle correlation effects. To perform the $\mathbf{k}$ sum above, we make use of the Hilbert transform [46] and rewrite Eq. (3) at each orbital and spin channel as

$$
G_{\sigma, a}^{\mathrm{loc}}(\omega)=\int d \epsilon \frac{\rho_{\sigma, a}^{(0)}(\epsilon)}{\omega-\Sigma_{\sigma, a}\left(\left[\mathcal{G}_{\sigma, a}^{0}(\omega)\right]\right)-\epsilon} .
$$


Here, $\rho_{\sigma, a}^{(0)}$ are the bare DOS of the carbon quantum dot displayed in Fig. 3 and $\mathcal{G}_{a, \sigma}^{0}(\omega)$ are the site-excluded Green's functions, usually referred to as Weiss functions [46]. Given the fact that we use diagonalized one-particle spectral functions for DMFT, computation of Eq. (4) can be performed for all local spin- and orbital-resolved Green's functions as well as for the corresponding bath propagators

$$
\left[\mathcal{G}_{\sigma, a}^{0}(\omega)\right]^{-1}=\frac{1}{G_{\sigma, a}^{\text {loc }}(\omega)}+\Sigma_{\sigma, a}(\omega) .
$$

These site-excluded Green's functions are used to compute self-consistently the multiorbital self-energies within our perturbative many-body scheme [47]. Equations (4) and (5) form a closed set of coupled, nonlinear relations which are solved numerically until convergence is achieved. Noteworthy, the equations above are reduced to the usual DFT+DMFT treatment, if the central atom is replaced by a single site in the lattice problem [48]. To obtain the self-energy of the carbon quantum dot we use multiorbital iterated perturbation theory (MO-IPT) as the impurity solver. The detailed formulation of MO-IPT for correlated electron systems has already been developed [47] and used in the context of carbon-based materials, $[14,40]$, so we do not repeat the equations here.

In what follows, we discuss our GGA+DMFT results. To pinpoint the relevance of MO electronic interactions in atomistic carbon quantum dots, we present in Fig. 4 the results for three different values of $U$ and fixed $J_{H}=0.4 \mathrm{eV}$. (This choice for $J_{H}$ is in accordance with values estimated in Ref. [49] for a different local moment problem in graphene.)

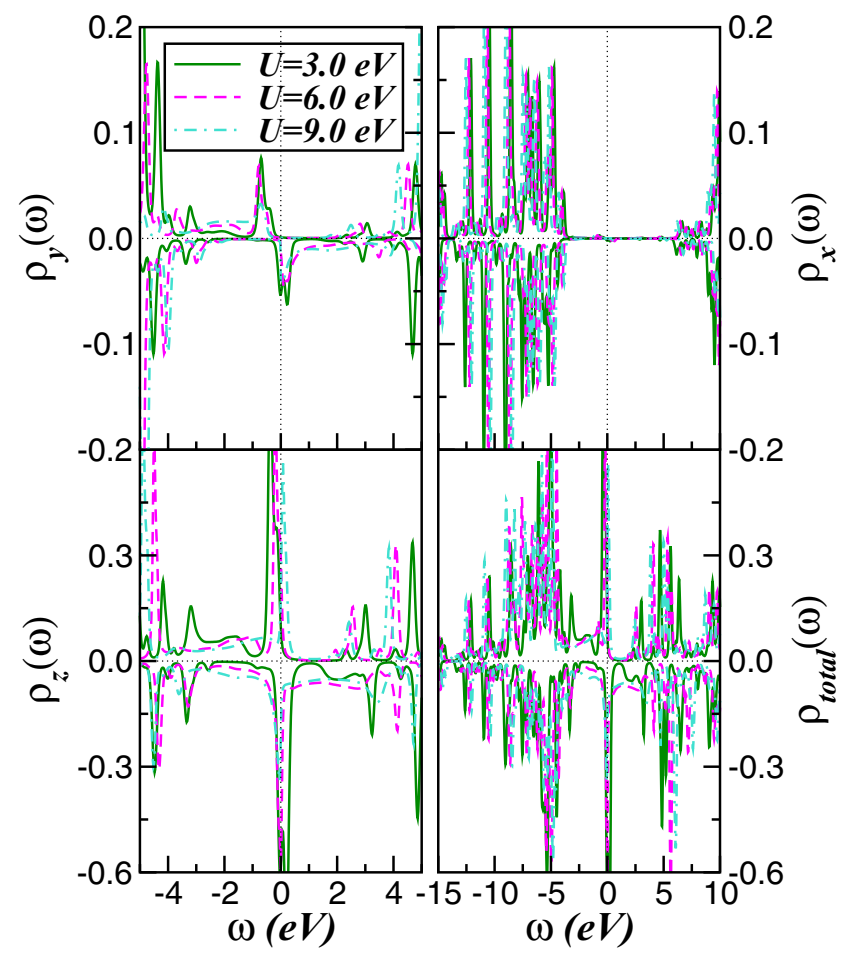

FIG. 4. GGA+DMFT results for electronic states of our carbon quantum dot, as a function of the on-site Coulomb repulsion $U$, for fixed $J_{H}=0.4 \mathrm{eV}$. Notice the evolution of the orbital-resolved DOS with increasing $U$ and the formation of pronounced Kondo clouds near $E_{F}$ in the $p_{z}$ orbital.
To trace the effect of electron-electron interactions, e.g., the appearance of channel-selective Kondo clouds at low energies, in Fig. 4 we show the evolution of the spin-orbitally resolved and total DOS with increasing $U$. One immediately notices that multiorbital electron-electron interactions modify the bare GGA spectral functions. As common to correlated electron systems, multiorbital dynamical correlations arising from $U, U^{\prime}$ and $J_{H}$ lead to spectral weight redistribution over large energy scales and an orbital-selective suppression of the one-particle band gap at low energies. While the semiconducting $p_{x}$ orbital is weakly affected by multiorbital electronic interactions, remarkable differences in the spectral weight transfer are found within the $p_{z}$ and $p_{y}$ channels. For the $p_{z}$ orbital, pronounced Kondo quasiparticles are visible at low energies and they are reshaped with increasing $U$. In this metallic channel two prominent shoulder features are resolved at $\omega \simeq-1.8 \mathrm{eV}$ (spin- $\uparrow$ DOS) and $\omega \simeq 2.0 \mathrm{eV}$ (spin- $\downarrow$ DOS), suggesting the formation of small Hubbard bands in this orbital. Spectral weight transfer is also found within the planar $p_{y}$ orbital; see Fig. 5 for details. Interestingly, while the spin- $\uparrow$ DOS shows insulating behavior, the conduction band in GGA of the spin- $\downarrow$ channel is transferred to low energies and reaches the Fermi energy for all values of $U$, as shown in Figs. 4 and 5. Apart from the visible spectral weight transfer at high energies, the overall spectral line shape in this orbital sector remains close to one found in GGA. However, the presence of spin- $\downarrow$ electronic states at $E_{F}$ suggests half-metallicity, which could be relevant for future spintronic devices. Our results for the $p_{y}$ orbital are consistent

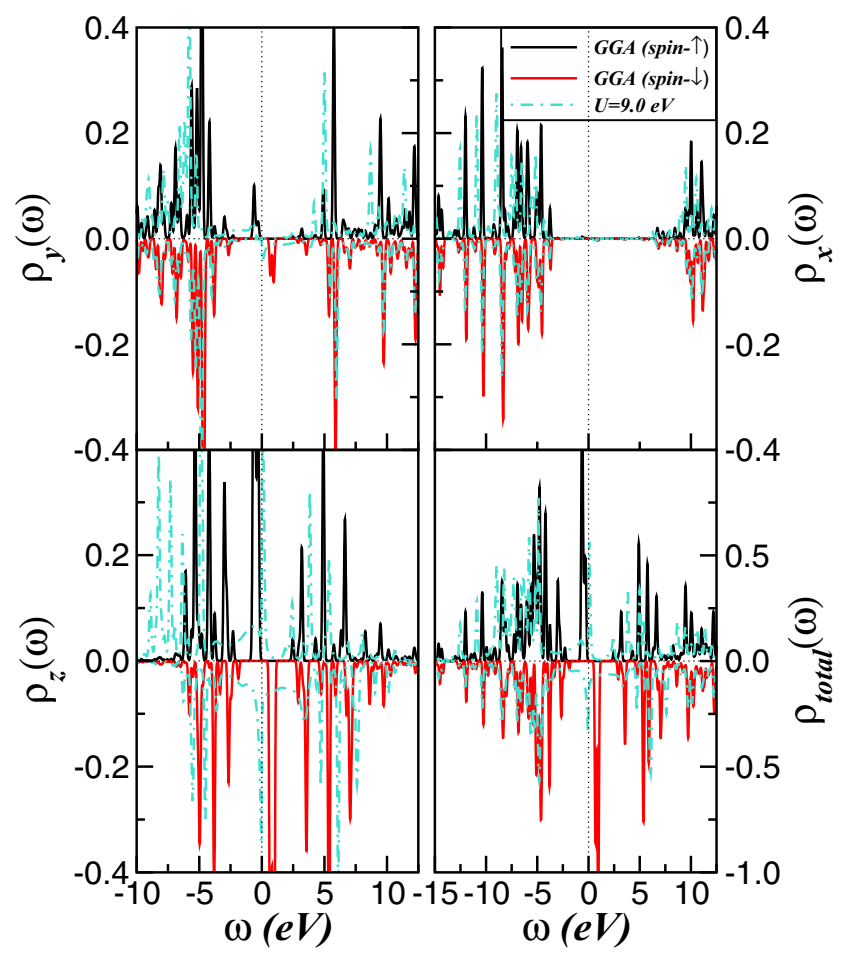

FIG. 5. Comparison between GGA and GGA+DMFT results for the electronic states of our carbon quantum dot. Notice that large-scale spectral weight transfer of the spin-resolved $p_{y}, p_{z}$ GGA+DMFT spectral functions and total DOS is obtained using realistic values [21] for the on-site Coulomb interaction $U$. 
with a recent inhomogeneous cluster-DMFT study on the graphene-lattice Hubbard model showing the coexistence of pseudogapped (spin- $\uparrow$ ) and Kondo quasiparticle (spin- $\downarrow$ ) electronic states near $E_{F}$ [50]. Taken together, at finite but not extreme $U$, our carbon quantum dot is on the Kondo side of the correlated phase diagram of nanoengineered graphene. Future spin-resolved scanning tunneling microscopy (STM) and spectroscopy (STS) experiments $[13,51]$ are called for to corroborate this prediction.

\section{CONCLUSION}

In conclusion, we propose to use an atomistically engineered nanostructure made of functionalized graphene nanoflakes to reveal metallic Kondo clouds in a carbon quantum dot. The geometry we propose is important for understanding the basic principles of edge and tunable magnetism in nanostructures made of monolayer graphene. To shed light onto this problem, we first performed first-principles GGA calculations to confirm the localized and magnetically ordered phase of functionalized graphene nanoflakes. We further employed GGA+DMFT for a multiband Hubbard model to explore the intrinsic correlated nature of the channelselective metallic phase of a carbon quantum dot. Our results suggest close underlying similarities (in spite of the different chemistry) between the Kondo regime emerging here and the Kondo resonance peaks induced by transition-metal adatoms in graphene [51]. Our theory is of fundamental importance to low-dimensional systems where multiorbital dynamical correlations induce the coexistence of semiconducting and (semi)-metallic regimes in spintronic materials.

\section{ACKNOWLEDGMENTS}

L.C.'s work is supported by CNPq (Proc. No. 307487/20148). Acknowledgment (L.C.) is also made to G. Seifert for discussions and the Department of Theoretical Chemistry at Technical University Dresden for hospitality. T.A.S.P. thanks PRONEX/CNPq/FAPEMAT 850109/2009 for financial support. M.V.M. acknowledges support from Research Foundation-Flanders (FWO), TOPBOF, and the CAPES-PVE program.
[1] K. S. Novoselov, A. K. Geim, S. V. Morozov, D. Jiang, Y. Zhang, S. V. Dubonos, I. V. Grigorieva1, and A. A. Firsov, Science 306, 666 (2004).

[2] See, D. R. Cooper, B. D’Anjou, N. Ghattamaneni, B. Harack, M. Hilke, A. Horth, N. Majlis, M. Massicotte, L. Vandsburger, E. Whiteway, and V. Yu, arXiv:1110.6557, and references therein.

[3] J. Hong, E. Bekyarova, P. Liang, W. A. de Heer, R. C. Haddon, and S. Khizroev, Sci. Rep. 2, 624 (2012).

[4] Y. Wu, Y.-M. Lin, A. A. Bol, K. A. Jenkins, F. Xia, D. B. Farmer, Y. Zhu, and P. Avouris, Nature (London) 472, 74 (2011); see also, Y.-M. Lin, C. Dimitrakopoulos, K. A. Jenkins, D. B. Farmer, H.-Y. Chiu, A. Grill, and Ph. Avouris, Science 327, 662 (2010).

[5] Y. W. Son, M. L. Cohen, and S. G. Louie, Nature (London) 444, 347 (2006).

[6] J. Nilsson, A. H. Castro Neto, F. Guinea, and N. M. R. Peres, Phys. Rev. B 76, 165416 (2007).

[7] A. A. Balandin, Nat. Mater. 10, 569 (2011); C. Berger, Z. Song, X. Li, X. Wu, N. Brown, C. Naud, D. Mayou, T. Li, J. Hass, A. N. Marchenkov, E. H. Conrad, P. N. First, and W. A. de Heer, Science 312, 1191 (2006); K. S. Novoselov, D. Jiang, T. Booth, V. V. Khotkevich, S. M. Morozov, and A. K. Geim, PNAS 102, 10451 (2005).

[8] D. Pesin and A. H. MacDonald, Nat. Mater. 11, 409 (2012).

[9] T. Jungwirth, J. Sinova, J. Mašek, J. Kučera, and A. H. MacDonald, Rev. Mod. Phys. 78, 809 (2006).

[10] A. H. Castro Neto, F. Guinea, N. M. R. Peres, K. S. Novoselov, and A. K. Geim, Rev. Mod. Phys. 81, 109 (2009).

[11] M. Fujita, K. Wakabayashi, K. Nakada, and K. Kusakabe, J. Phys. Soc. Jpn. 65, 1920 (1996).

[12] M. Golor, C. Koop, T. C. Lang, S. Wessel, and M. J. Schmidt, Phys. Rev. Lett. 111, 085504 (2013), and references therein.

[13] C. Tao, L. Jiao, O. V. Yazyev, Y.-C. Chen, J. Feng, X. Zhang, R. B. Capaz, J. M. Tour, A. Zettl, S. G. Louie, H. Dai, and M. F. Crommie, Nat. Phys. 7, 616 (2011).

[14] L. Craco, D. Selli, G. Seifert, and S. Leoni, Phys. Rev. B 91, 205120 (2015).
[15] R. L. Frank and E. H. Lieb, Phys. Rev. Lett. 107, 066801 (2011).

[16] P. Durand, G. R. Darling, Y. Dubitsky, A. Zaopo, and M. J. Rosseinsky, Nat. Mater. 2, 605 (2003).

[17] J. A. Chan, S. Lany, and A. Zunger, Phys. Rev. Lett. 103, 016404 (2009); J. Winterlik, G. H. Fecher, C. A. Jenkins, C. Felser, C. Mühle, K. Doll, M. Jansen, L. M. Sandratskii, and Jürgen Kübler, ibid. 102, 016401 (2009); A. J. R. da Silva and L. M. Falicov, Phys. Rev. B 52, 2325 (1995); L. Craco and S. Leoni, Sci. Rep. 5, 13772 (2015).

[18] G. Chiappe, E. Louis, E. SanFabián, and J. A. Verges, Phys. Rev. B 75, 195104 (2007).

[19] M. Imada, A. Fujimori, and Y. Tokura, Rev. Mod. Phys. 70, 1039 (1998).

[20] V. N. Kotov, B. Uchoa, V. M. Pereira, F. Guinea, and A. H. Castro Neto, Rev. Mod. Phys. 84, 1067 (2012).

[21] T. O. Wehling, E. Şaşığlu, C. Friedrich, A. I. Lichtenstein, M. I. Katsnelson, and S. Blügel, Phys. Rev. Lett. 106, 236805 (2011).

[22] K. Sengupta and G. Baskaran, Phys. Rev. B 77, 045417 (2008), and references therein.

[23] R. Bulla, T. A. Costi, and T. Pruschke, Rev. Mod. Phys. 80, 395 (2008).

[24] P. Jacobson, T. Herden, M. Muenks, G. Laskin, O. Brovko, V. Stepanyuk, M. Ternes, and K. Kern, Nat. Commun. 6, 8536 (2015).

[25] M. Grobis, I. G. Rau, R. M. Potok, and D. GoldhaberGordon, Handbook of Magnetism and Advanced Magnetic Materials (Wiley, New York, 2007), Vol. 5; see also, arXiv:cond-mat/0611480.

[26] G. Granger, M. A. Kastner, I. Radu, M. P. Hanson, and A. C. Gossard, Phys. Rev. B 72, 165309 (2005).

[27] Y. Ominato and M. Koshino, Phys. Rev. B 87, 115433 (2013).

[28] P. Potasz, A. D. Güçlü, A. Wójs, and P. Hawrylak, Phys. Rev. B 85, 075431 (2012); see also, P. Potasz, A. D. Güçlü, O. Voznyy, J. A. Folk, and P. Hawrylak, ibid. 83, 174441 (2011).

[29] X. Li, J. Zhou, Q. Wang, X. Chen, Y. Kawazoe, and P. Jena, New J. Phys. 14, 033043 (2012). 
[30] J. Zhou, Q. Wang, Q. Sun, and P. Jena, Phys. Rev. B 84, 081402 (2011).

[31] A. Valli, H. Das, G. Sangiovanni, T. Saha-Dasgupta, and K. Held, Phys. Rev. B 92, 115143 (2015).

[32] J. M. Soler, E. Artacho, J. D. Gale, A. García, J. Junquera, P. Ordejón, and D. Sánchez-Portal, J. Phys. Condens. Matter 14, 2745 (2002); E. Artacho, E. Anglada, O. Dieguez, J. D. Gale, A. García, J. Junquera, M. Martin, P. Ordejón, J. M. Pruneda, D. Sánchez-Portal, and J. M. Soler, ibid. 20, 064208 (2008).

[33] H. J. Monkhorst and J. D. Pack, Phys. Rev. B 13, 5188 (1976).

[34] N. Troullier and J. L. Martins, Phys. Rev. B 43, 1993 (1991).

[35] J. P. Perdew, K. Burke, and M. Ernzerhof, Phys. Rev. Lett. 77, 3865 (1996).

[36] W. Kohn and L. J. Sham, Phys. Rev. 140, A1133 (1965).

[37] J. Junquera, Ó. Paz, D. Sánchez-Portal, and E. Artacho, Phys. Rev. B 64, 235111 (2001).

[38] J. Moreno and J. M. Soler, Phys. Rev. B 45, 13891 (1992).

[39] S. Zhang, J. Zhou, X. Li, and Q. Wang, J. Nanopart. Res. 14, 1171 (2012).

[40] L. Craco, M. S. Laad, S. Leoni, and A. S. de Arruda, Phys. Rev. B 87, 155109 (2013).
[41] X. Li and Q. Wang, Phys. Chem. Chem. Phys. 14, 2065 (2012).

[42] Y. Kobayashi, K.-i. Fukui, T. Enoki, K. Kusakabe, and Y. Kaburagi, Phys. Rev. B 71, 193406 (2005).

[43] E. H. Lieb, Phys. Rev. Lett. 62, 1201 (1989).

[44] Notice that in $H_{\text {int }}$ we have singed out all remaining atoms, retaining explicit multiorbital electron-electron interactions only in the central carbon quantum dot.

[45] R. Peters and N. Kawakami, Phys. Rev. B 92, 075103 (2015).

[46] A. Georges, G. Kotliar, W. Krauth, and M. J. Rozenberg, Rev. Mod. Phys. 68, 13 (1996).

[47] L. Craco, Phys. Rev. B 77, 125122 (2008).

[48] G. Kotliar, S. Y. Savrasov, K. Haule, V. S. Oudovenko, O. Parcollet, and C. A. Marianetti, Rev. Mod. Phys. 78, 865 (2006).

[49] M. Casartelli, S. Casolo, G. F. Tantardini, and R. Martinazzo, Phys. Rev. B 88, 195424 (2013).

[50] M. Charlebois, D. Sénéchal, A.-M. Gagnon, and A.-M. S. Tremblay, Phys. Rev. B 91, 035132 (2015).

[51] J. Ren, H. Guo, J. Pan, Y. Y. Zhang, X. Wu, H.-G. Luo, S. Du, S. T. Pantelides, and H.-J. Gao, Nano Lett. 14, 4011 (2014); L. Feng et al., Appl. Phys. Lett. 101, 113113 (2012). 